\title{
Biogenic hydrogen and methane production from Chlorella vulgaris and Dunaliella tertiolecta biomass
}

Aino-Maija Lakaniemi ${ }^{1 *}$, Christopher J Hulatt ${ }^{2}$, David N Thomas ${ }^{2,3}$, Olli H Tuovinen ${ }^{1,4}$ and Jaakko A Puhakka

\begin{abstract}
Background: Microalgae are a promising feedstock for biofuel and bioenergy production due to their high photosynthetic efficiencies, high growth rates and no need for external organic carbon supply. In this study, utilization of Chlorella vulgaris (a fresh water microalga) and Dunaliella tertiolecta (a marine microalga) biomass was tested as a feedstock for anaerobic $\mathrm{H}_{2}$ and $\mathrm{CH}_{4}$ production.

Results: Anaerobic serum bottle assays were conducted at $37^{\circ} \mathrm{C}$ with enrichment cultures derived from municipal anaerobic digester sludge. Low levels of $\mathrm{H}_{2}$ were produced by anaerobic enrichment cultures, but $\mathrm{H}_{2}$ was subsequently consumed even in the presence of 2-bromoethanesulfonic acid, an inhibitor of methanogens. Without inoculation, algal biomass still produced $\mathrm{H}_{2}$ due to the activities of satellite bacteria associated with algal cultures. $\mathrm{CH}_{4}$ was produced from both types of biomass with anaerobic enrichments. Polymerase chain reactiondenaturing gradient gel electrophoresis profiling indicated the presence of $\mathrm{H}_{2}$-producing and $\mathrm{H}_{2}$-consuming bacteria in the anaerobic enrichment cultures and the presence of $\mathrm{H}_{2}$-producing bacteria among the satellite bacteria in both sources of algal biomass.

Conclusions: $\mathrm{H}_{2}$ production by the satellite bacteria was comparable from D. tertiolecta $(12.6 \mathrm{ml} \mathrm{H} / \mathrm{g}$ volatile solids (VS)) and from C. vulgaris (10.8 $\mathrm{ml} \mathrm{H} / \mathrm{g}$ VS), whereas $\mathrm{CH}_{4}$ production was significantly higher from C. vulgaris $(286 \mathrm{ml} / \mathrm{g}$ VS) than from D. tertiolecta $(24 \mathrm{ml} / \mathrm{g}$ VS). The high salinity of the D. tertiolecta slurry, prohibitive to methanogens, was the probable reason for lower $\mathrm{CH}_{4}$ production.
\end{abstract}

\section{Background}

Photosynthetic biomass-based fuels are widely considered as viable contenders as sustainable alternatives to fossil fuels. Currently, the major share of biofuels and other forms of bioenergy are produced from terrestrial plants [1]. Microalgae may prove an alternative to terrestrial crops because they have higher photosynthetic efficiencies, higher yields and growth rates, and fewer requirements for cultivation land and they can be grown in saline waters and in arid and barren land areas [1,2]. Microalgal biomass is potent for anaerobic conversion as it can have a high content of lipids, carbohydrates and proteins, and does not contain recalcitrant lignin

\footnotetext{
* Correspondence: aino-maija.lakaniemi@tut.fi

'Department of Chemistry and Bioengineering, Tampere University of

Technology, PO Box 541, Fl-33101 Tampere, Finland

Full list of author information is available at the end of the article
}

[1-3]. However, the robust cell walls of some microalgal species may limit digestibility $[4,5]$.

Anaerobic digestion of microalgal biomass for $\mathrm{CH}_{4}$ production has been studied at various temperatures and with various pretreatments and cosubstrates [4,6-9]. For example, Chen and Oswald [4] reported that pretreatment of algal biomass at $100^{\circ} \mathrm{C}$ for $8 \mathrm{~h}$ increased digestibility by up to $33 \%$, but the energy consumed in pretreatment was higher than the enhancement gained in $\mathrm{CH}_{4}$ production [8].

Some green microalgae, such as Chlamydomonas reinhardtii [10] and Chlorella salina [11] produce hydrogen under anaerobic conditions via direct photolysis [12]. However, despite extensive research this process has low yields and is rather feeble. It is filled with metabolic and technical obstacles [13] and remains an unlikely source of sustainable energy. Indirect photolysis of microalgal biomass by first hydrolyzing the biomass with lactic acid
C Biomed Central 
bacteria followed by photosynthetic $\mathrm{H}_{2}$ production resulted in $\mathrm{H}_{2}$ yields up to $8 \mathrm{~mol} \mathrm{H}_{2} / \mathrm{mol}$ starch glucose from $C$. reinhardtii (66\% starch conversion efficiency) [14]. Carver et al. [5] reported $\mathrm{H}_{2}$ production from dark fermentation of Chlorella vulgaris and Dunaliella tertiolecta at $60^{\circ} \mathrm{C}$. Further, Gfeller and Gibbs [15], Miura et $a l$. [16] and Ueno et al. [17] reported hydrogen fermentation by microalgal cells under dark, anaerobic conditions.

The aim of this study was to examine the formation of $\mathrm{H}_{2}$ and $\mathrm{CH}_{4}$ from microalgal biomass. Two green microalgae, Chlorella vulgaris (a freshwater species) and Dunaliella tertiolecta (a marine species) were used as feedstocks. Experiments were carried out in batch bottles at $37^{\circ} \mathrm{C}$ without pretreatment of the algal biomass, and the microbial communities were characterized by polymerase chain reaction-denaturing gradient gel electrophoresis (PCR-DGGE) profiling of 16S rRNA gene and sequencing.

\section{Results}

\section{Algal biomass feedstocks}

The chemical composition of the two microalgal biomass feedstocks was different. C. vulgaris contained $36 \%, 13 \%$ and $8 \%$ of proteins, lipids and sugars on a dry weight basis, respectively. The corresponding mass composition of $D$. tertiolecta was $15 \%, 11 \%$ and $4 \%$, respectively. In general, these values are lower than previously reported in the literature (Additional file 1, Table S1). The compositional data for $D$. tertiolecta in particular may reflect loss of cellular constituents upon sample preparation and handling because the marine microalga does not have a rigid wall and is prone to lyse when the osmotic pressure changes. Growth conditions were not varied to determine the corresponding changes in cellular fractions.

\section{Enrichment cultures}

Four different cultures were enriched from the initial anaerobic digester sludge. Two $\mathrm{H}_{2}$-fermenting cultures, one with C. vulgaris biomass, designated as B-C, and one with $D$. tertiolecta biomass as the substrate, B-D, and two $\mathrm{CH}_{4}$-producing cultures, one utilizing C. vulgaris biomass, U-C, and one $D$. tertiolecta biomass, UD. Methanogenesis was suppressed in the $\mathrm{H}_{2}$-fermenting cultures by addition of $20 \mathrm{mM}$ 2-bromoethanesulfonic acid (BESA). During enrichment phases 1-5 no $\mathrm{H}_{2}$ was produced in any of the cultures, while in enrichment phases 6 to 9 low levels of $\mathrm{H}_{2}$ were detected in $\mathrm{B}-\mathrm{C}$ and $\mathrm{B}-\mathrm{D}$ enrichments during the first few days, but usually by day 5 the $\mathrm{H}_{2}$ level had decreased below detection limit (results not shown). No $\mathrm{CH}_{4}$ was produced in the cultures with added BESA (Figure 1).
With U-C and U-D, the $\mathrm{CH}_{4}$ production was higher from $D$. tertiolecta biomass than from $C$. vulgaris biomass in the first enrichment phase when tested with a combination of $25 \%$ algal biomass and $75 \%$ activated sludge (Figure 1A). From phase 2 onwards, when the proportion of algal biomass in the substrate was increased to $50 \%$ or higher, $\mathrm{CH}_{4}$ production from $C$. vulgaris surpassed that from $D$. tertiolecta (Figure 1B-F). With $100 \%$ C. vulgaris and D. tertiolecta biomass the rates of $\mathrm{CH}_{4}$ production ranged between 3.4-6.5 and 1.2-4.9 $\mathrm{ml} /$ day and the lag times between 2.6-5.1 and 5.3-10 days, respectively. The $\mathrm{CH}_{4}$ yield and $\mathrm{CH}_{4}$ production rate decreased and the lag time increased from D. tertiolecta as the enrichment proceeded. The $\mathrm{CH}_{4}$ yields from $C$. vulgaris remained more or less constant after enrichment phase 4 (Figure 1).

\section{$\mathrm{H}_{2}$ and $\mathrm{CH}_{4}$ production potential}

Gas production potential from C. vulgaris and D. tertiolecta was studied using the enrichment cultures after nine passages. Some $\mathrm{CO}_{2}$ was produced in all bottles indicating degradation in all cultures, including all controls with no anaerobic inoculum (Table 1, Figures 2B and $3 \mathrm{~B}) . \mathrm{CO}_{2}$ production was higher from $C$. vulgaris compared to $D$. tertiolecta.

$\mathrm{H}_{2}$ was produced in all cultures including the controls on day 1 . With glucose in particular, high levels of $\mathrm{H}_{2}$ were produced during first few days. Over time $\mathrm{H}_{2}$ decreased to undetectable levels in all cultures except those with algal biomass without inoculum and cultures with glucose and B-D. In the other cultures $\mathrm{H}_{2}$ was consumed due to interspecies $\mathrm{H}_{2}$ transfer, and cumulative $\mathrm{H}_{2}$ production from algal biomass with the anaerobic inocula was negligible (Table 1 ). With no anaerobic inoculum, $\mathrm{H}_{2}$ production was higher from $D$. tertiolecta biomass, 8.4 and $12.6 \mathrm{ml} \mathrm{H}_{2} / \mathrm{g}$ volatile solids (VS), than from C. vulgaris biomass, 7.9 and $10.8 \mathrm{ml} \mathrm{H}_{2} / \mathrm{g}$ VS, with and without BESA, respectively. Further enhancement of $\mathrm{H}_{2}$ production was attempted by using these cultures as inoculum in batch incubations, but after four enrichment steps no increase in $\mathrm{H}_{2}$ production was detected.

No $\mathrm{CH}_{4}$ was produced in the cultures amended with BESA (Figure 2). A significant amount of $\mathrm{CH}_{4}$ was produced only with $C$. vulgaris and $\mathrm{U}-\mathrm{C}$, glucose and $\mathrm{U}-\mathrm{C}$, and glucose and U-D (Table 1). Some $\mathrm{CH}_{4}$ was also produced with $D$. tertiolecta and U-D as well as with chitosan and U-C (Table 1). $\mathrm{CH}_{4}$ production from cellulose was negligible. $\mathrm{CH}_{4}$ production from chitosan was significantly lower than that from microalgal biomass. Gas production in controls with no substrate but inoculum was very low, and was taken into account in calculation of the gas production yields (Table 2). Thus, $\mathrm{CH}_{4}$ was produced from both $C$. vulgaris and D. tertiolecta biomass, while the yield was substantially lower with $D$. 


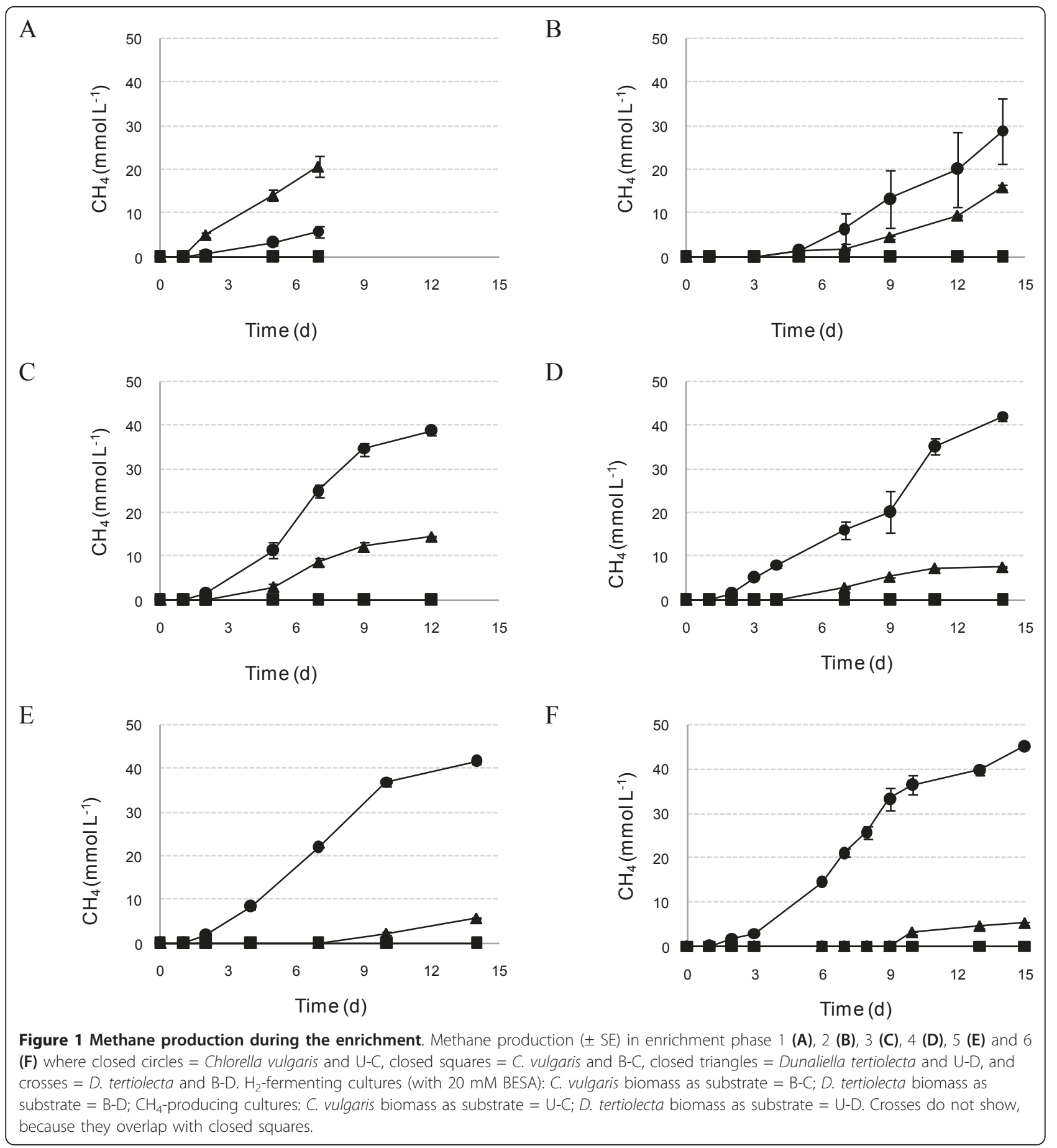

tertiolecta than with C. vulgaris (Table 2). With C. vulgaris biomass $30.6 \%$ of organic carbon was released as $\mathrm{CH}_{4}$ and $13.6 \%$ as $\mathrm{CO}_{2}$, while with $D$. tertiolecta biomass the corresponding values were 5.2 and $2.6 \%$, respectively. $\mathrm{CH}_{4}$ production from $C$. vulgaris biomass was higher than in glucose controls, while $\mathrm{CH}_{4}$ production from $D$. tertiolecta remained far below that of the glucose controls. With glucose, cellulose or chitosan, the
$\mathrm{H}_{2}$ production was generally higher with the B-D enrichment than with the $\mathrm{B}-\mathrm{C}$ enrichment, but $\mathrm{CH}_{4}$ production was generally higher with the $\mathrm{U}-\mathrm{C}$ enrichment than with the U-D enrichment (Table 1).

The average chloride ion concentration in the anaerobic incubations was 0.7 and $4.8 \mathrm{~g} / \mathrm{l}$ and sodium ion concentration was 2.3 and $2.1 \mathrm{~g} / \mathrm{l}$ in bottles with C. vulgaris and $D$. tertiolecta as the substrate, respectively. The $\mathrm{pH}$ 
Table 1 Metabolite production in all cultures: cumulative gas production and accumulation of metabolites in the test cultures after 49 day of incubation

\begin{tabular}{|c|c|c|c|c|c|}
\hline Substrate & Inoculum & $\mathrm{H}_{2}(\mathrm{ml})$ & $\mathrm{CH}_{4}(\mathrm{ml})$ & $\mathrm{CO}_{2}(\mathrm{ml})$ & Sum of VFA and alcohols (mM) \\
\hline None & U-C & $0.0 \pm 0.0$ & $0.2 \pm 0.3$ & $2.3 \pm 0.4$ & $-0.6 \pm 0.7$ \\
\hline None & $B-C$ & $0.0 \pm 0.0$ & $0.0 \pm 0.0$ & $4.7 \pm 0.2$ & $5.1 \pm 0.5$ \\
\hline None & $U-D$ & $0.0 \pm 0.0$ & $0.0 \pm 0.0$ & $1.8 \pm 0.0$ & $-0.5 \pm 0.1$ \\
\hline None & $B-D$ & $0.0 \pm 0.0$ & $0.0 \pm 0.0$ & $4.5 \pm 0.2$ & $3.7 \pm 0.4$ \\
\hline Chlorella vulgaris & None & $2.1 \pm 0.7$ & $0.0 \pm 0.0$ & $10.8 \pm 0.6$ & $22.1 \pm 1.8$ \\
\hline C. vulgaris and $\mathrm{BESA}$ & None & $1.3 \pm 0.2$ & $0.0 \pm 0.0$ & $12.0 \pm 0.7$ & $19.1 \pm 5.1$ \\
\hline Dunaliella tertiolecta & None & $2.8 \pm 0.1$ & $0.0 \pm 0.0$ & $3.0 \pm 0.4$ & $5.1 \pm 0.1$ \\
\hline D. tertiolecta and BESA & None & $1.5 \pm 0.3$ & $0.0 \pm 0.0$ & $4.2 \pm 0.2$ & $4.0 \pm 0.3$ \\
\hline C. vulgaris & $U-C$ & $0.0 \pm 0.0$ & $74.9 \pm 3.6$ & $35.2 \pm 0.3$ & $-3.8 \pm 1.0$ \\
\hline C. vulgaris & $B-C$ & $0.1 \pm 0.0$ & $0.0 \pm 0.0$ & $24.8 \pm 0.0$ & $31.2 \pm 0.7$ \\
\hline D. tertiolecta & U-D & $0.0 \pm 0.0$ & $4.7 \pm 0.2$ & $4.9 \pm 0.0$ & $0.4 \pm 0.1$ \\
\hline D. tertiolecta & B-D & $0.0 \pm 0.0$ & $0.0 \pm 0.0$ & $7.4 \pm 0.8$ & $8.9 \pm 0.4$ \\
\hline Glucose & U-C & $4.9 \pm 0.4$ & $56.4 \pm 0.1$ & $62.5 \pm 0.4$ & $2.9 \pm 0.7$ \\
\hline Glucose & $B-C$ & $7.1 \pm 0.4$ & $0.0 \pm 0.0$ & $57.9 \pm 0.9$ & $46.1 \pm 0.4$ \\
\hline Glucose & U-D & $5.2 \pm 0.9$ & $38.5 \pm 14.0$ & $56.2 \pm 7.0$ & $13.3 \pm 14.9$ \\
\hline Glucose & $B-D$ & $14.6 \pm 2.3$ & $0.0 \pm 0.0$ & $60.8 \pm 0.5$ & $44.4 \pm 7.1$ \\
\hline Cellulose & U-C & $0.0 \pm 0.0$ & $0.3 \pm 0.4$ & $0.9 \pm 0.1$ & $0.7 \pm 1.4$ \\
\hline Cellulose & $\mathrm{B}-\mathrm{C}$ & $0.0 \pm 0.0$ & $0.0 \pm 0.0$ & $4.0 \pm 0.0$ & $7.6 \pm 0.4$ \\
\hline Cellulose & U-D & $0.0 \pm 0.0$ & $0.1 \pm 0.1$ & $1.8 \pm 0.1$ & $0.0 \pm 0.1$ \\
\hline Cellulose & B-D & $0.0 \pm 0.0$ & $0.0 \pm 0.0$ & $4.6 \pm 0.3$ & $5.4 \pm 1.3$ \\
\hline Chitosan & $U-C$ & $0.0 \pm 0.0$ & $3.6 \pm 4.1$ & $2.4 \pm 2.5$ & $0.4 \pm 1.5$ \\
\hline Chitosan & $B-C$ & $0.0 \pm 0.0$ & $0.0 \pm 0.0$ & $3.1 \pm 0.3$ & $6.9 \pm 0.5$ \\
\hline Chitosan & U-D & $0.0 \pm 0.0$ & $0.0 \pm 0.0$ & $1.0 \pm 0.0$ & $0.1 \pm 0.1$ \\
\hline Chitosan & B-D & $0.0 \pm 0.0$ & $0.0 \pm 0.0$ & $3.2 \pm 0.1$ & $5.6 \pm 0.8$ \\
\hline
\end{tabular}

A minus sign in front of sum of VFA and alcohols indicates that the sum of VFA and alcohols was higher on day 0 than on day 49 . The values include standard errors.

$\mathrm{H}_{2}$-fermenting cultures: $\mathrm{C}$. vulgaris biomass as substrate $=\mathrm{B}-\mathrm{C} ; \mathrm{D}$. tertiolecta biomass as substrate $=\mathrm{B}-\mathrm{D} ; \mathrm{CH}_{4}$-producing cultures: $\mathrm{C}$. vulgaris biomass as substrate $=$ $\mathrm{U}-\mathrm{C} ; \mathrm{D}$. tertiolecta biomass as substrate $=\mathrm{U}-\mathrm{D}$.

$\mathrm{BESA}=2$-bromoethanesulfonic acid; VFA = volatile fatty acids.

of the medium was not adjusted at the beginning of the anaerobic incubation. The initial $\mathrm{pH}$ was 8.0 in the cultures with algal biomass and 8.5 with the other substrates and the cultures with no substrate. With no substrate, cellulose and chitosan the $\mathrm{pH}$ changes were minimal, $\mathrm{pH}$ ranging from $\mathrm{pH} 8.0$ to 8.5 during the incubation. With algal biomass, but no inoculum the $\mathrm{pH}$ varied between 7.5 and 8.0. With $C$. vulgaris and U-C the $\mathrm{pH}$ was 7.5-8.0, with $C$. vulgaris and B-C 7.0-8.0, with $D$. tertiolecta and U-D 8.0-8.5, and with D. tertiolecta and B-D 7.5-8.0. In cultures with glucose the $\mathrm{pH}$ varied between 6.0 and 8.5 .

Organic acids accumulated in the cultures with the B$\mathrm{C}$ and B-D enrichments as well as in the cultures with no anaerobic enrichment inoculum. In the cultures inoculated with U-C and U-D organic acids accumulated only at the beginning of the incubation and were later reduced to $\mathrm{CH}_{4}$ (Figure 2C,D). In some cultures, such as with $C$. vulgaris and $\mathrm{U}-\mathrm{C}$, the volatile fatty acids (VFA) and ethanol concentrations were lower on day 49 than on day 0 (Table 1 ). The total concentrations of the soluble degradation products were lower with $D$. tertiolecta than with $C$. vulgaris (Table 1 ). The main VFA in the anaerobic inocula were acetate and propionate (Figure $2 \mathrm{C}, \mathrm{D})$, and acetate and formate in the cultures with no inoculum (Figure 3C,D).

The initial total chemical oxygen demand $\left(\mathrm{COD}_{\text {tot }}\right)$ values were significantly higher in cultures with $C$. vulgaris than with $D$. tertiolecta in spite of identical initial concentrations of algal VS in all cultures. The addition of $20 \mathrm{mM}$ BESA also increased the initial COD concentration. The $\mathrm{COD}_{\text {tot }}$ concentrations decreased in all cultures between days 0 and 49, except in the case of no substrate and in cultures with $C$. vulgaris, BESA and no inoculum. The decrease in $\mathrm{COD}_{\text {tot }}$ was greater in bottles with U-C and U-D than with B-C and B-D as inoculum, respectively (Figure $4 \mathrm{~A}$ ). $\mathrm{COD}_{\text {tot }}$ reduction was $52 \%$ with C. vulgaris and U-C, and $57 \%$ D. tertiolecta and UD, but only $21 \%$ with C. vulgaris and B-C, and $15 \%$ with $D$. tertiolecta and B-D, respectively. The ratio of soluble $\mathrm{COD}\left(\mathrm{COD}_{\mathrm{s}}\right)$ to $\mathrm{COD}_{\text {tot }}$ decreased with $\mathrm{CH}_{4}$ production, but increased in the other cultures (Figure $4 \mathrm{~B}$ ). 


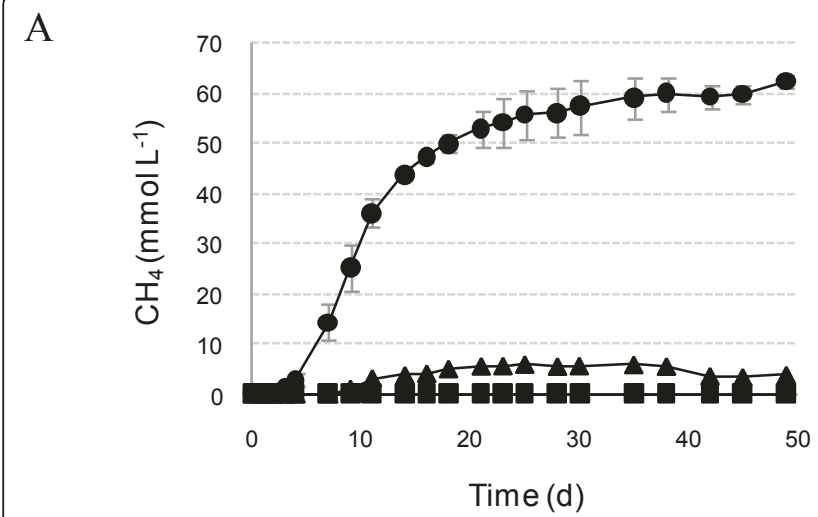

$\mathrm{C}$

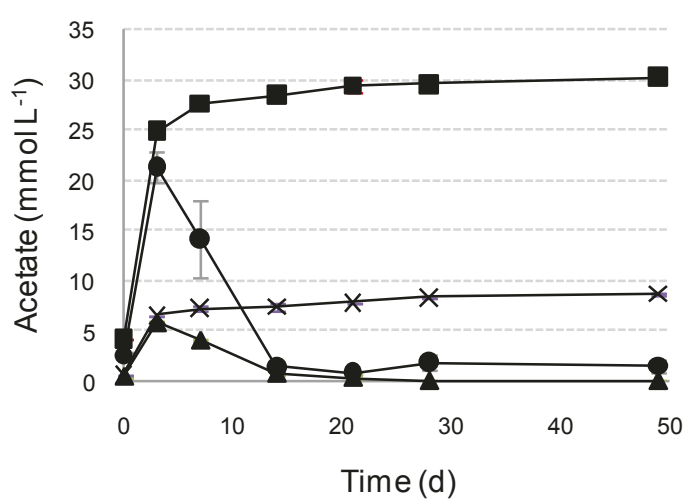

B

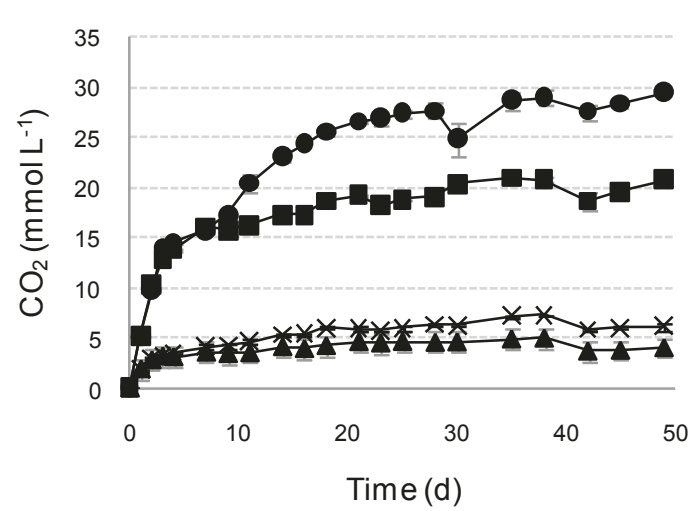

$\mathrm{D}$

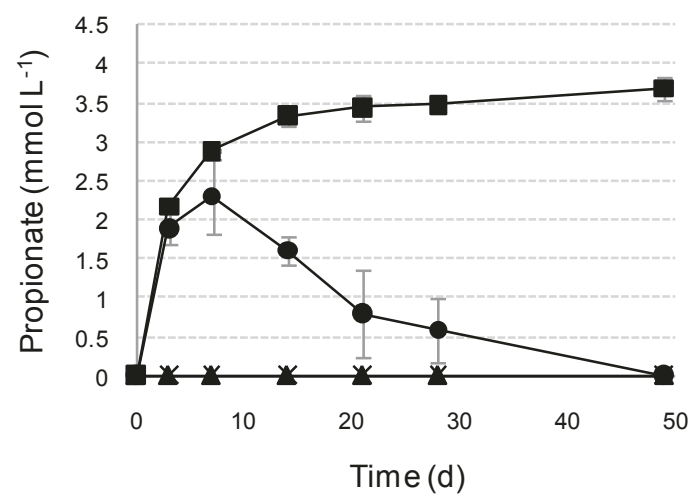

Figure 2 Metabolite production in cultures with the anaerobic enrichment inocula. $\mathrm{CH}_{4}(\mathbf{A}), \mathrm{CO}_{2}$ (B) and the main fermentation products acetate $(\mathbf{C})$ and propionate $(\mathbf{D})$ where closed circles $=$ Chlorella vulgaris and $\mathrm{U}-\mathrm{C}$, closed squares $=\mathrm{C}$. vulgaris and $\mathrm{B}-\mathrm{C}$, closed triangles $=$ Dunaliella tertiolecta and U-D, and crosses = D. tertiolecta and B-D. $\mathrm{H}_{2}$-fermenting cultures (with $20 \mathrm{mM} \mathrm{BESA}$ ): C. vulgaris biomass as substrate = $\mathrm{B}-\mathrm{C} ; \mathrm{D}$. tertiolecta biomass as substrate $=\mathrm{B}-\mathrm{D} ; \mathrm{CH}_{4}$-producing cultures: $\mathrm{C}$. vulgaris biomass as substrate $=\mathrm{U}-\mathrm{C} ; \mathrm{D}$. tertiolecta biomass as substrate $=$ U-D.

The COD results were in line with the VFA and alcohol results.

\section{Microbial community composition}

Based on bacterial PCR-DGGE and sequencing, the initial anaerobic inoculum contained bacteria belonging to phyla Firmicutes, Bacteroidetes, Proteobacteria and Chloroflexi (Additional file 2, Table S2). No genus and species level information for these bacterial sequences were obtained from GenBank.

The bacterial community became enriched during the ten serial batch incubations. Bacterial DGGE profiles were different with the two algal biomass types. The addition of BESA also affected the bacterial community composition (Figure 5). For example, bands B13 and B29 were only clear with C. vulgaris and B-C, but not with $C$. vulgaris and U-C. Further, bands B18 and B21 were only clear in C. vulgaris and U-C, but not with C. vulgaris and B-C. In addition, bands B30B33 were present in cultures with $D$. tertiolecta, but no corresponding bands were seen in cultures with C. vulgaris (Figure 5).

Most of the bacterial 16S rDNA sequences amplified from the anaerobic enrichments matched uncultured bacteria with no species-level information (Additional file 3, Table S3). The matches in the enrichments were Petrimonas spp. (band B14), Bacteroides spp. (B15), Bilophila wadsworthia (B26), Wolinella succinogenes (B34), Oceanibulbus indolifex (B35), and Syntrophobacter spp. (B39). Petrimonas spp. were present in all cultures with C. vulgaris and anaerobic inoculum, B. wadsworthia in C. vulgaris and B-C and Bacteroides spp. in C. vulgaris and B-C as well as in the duplicates of D. tertiolecta and U-D. W. succinogenes, O. indolifex and Syntrophobacter spp. were present in all cultures with $D$. tertiolecta and anaerobic inoculum (Figure 4).

A high diversity of bacteria was also present in cultures with no anaerobic inoculum (Figure 4 and Additional File 4, Table S4). These bacteria included Acidobacterium spp. (band B44), Clostridium spp. (B45, 


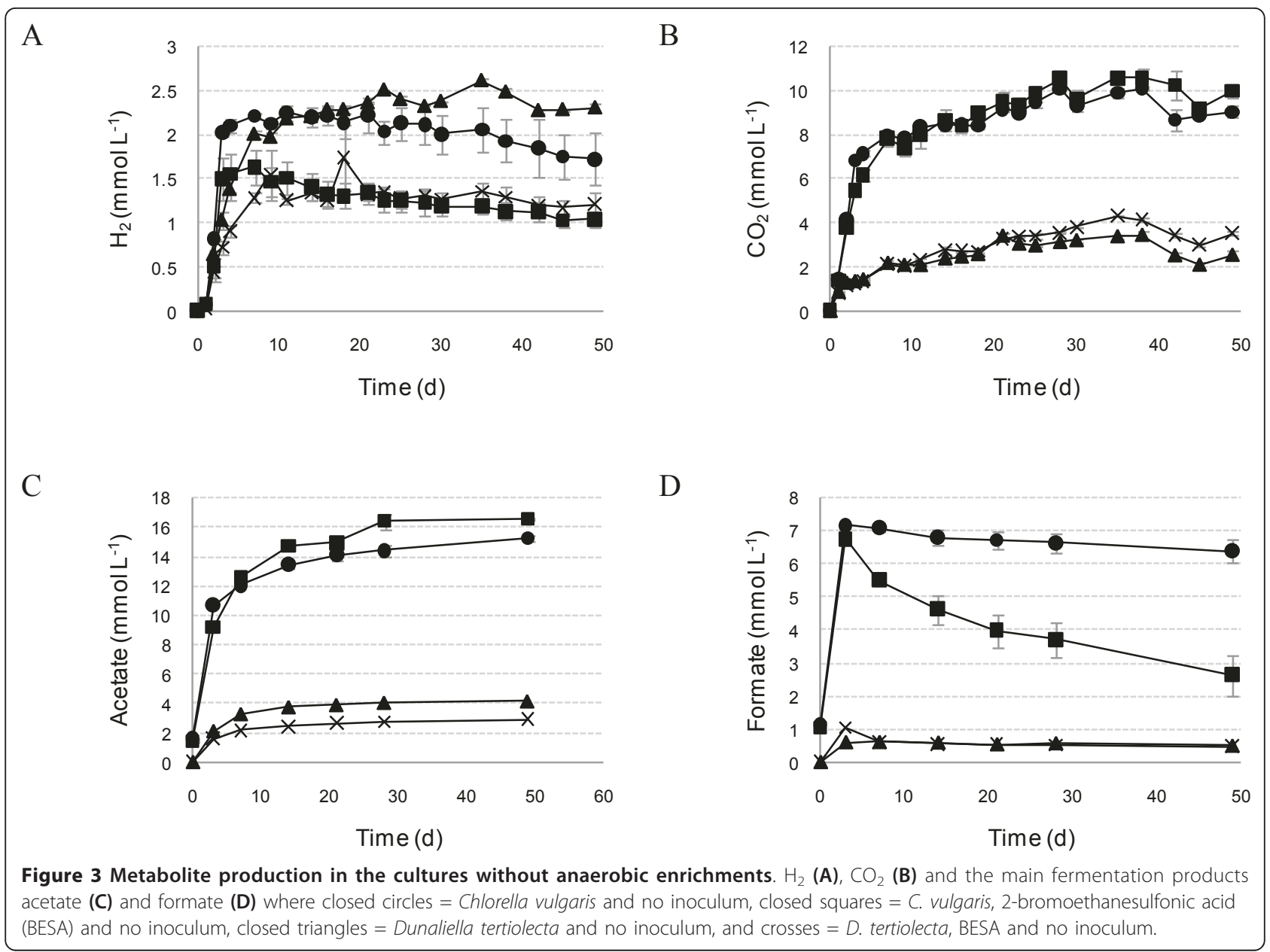

B46, B47, B61), Clostridium celerecrescens (B48, B63), Brevundimonas spp. (B49), Hafnia alvei (B50, B54), Hafnia alvei or Obesumbacterium proteus (B51), Gordonia terrae (B56), Clostridium sulfidigenes (B57, B58, B59,
B60), Oceanibulbus indolifex (B62), Roseobacter spp. (B65), Exiguobacterium spp. (B66), Bacillus thermoamylovorans (B67) and four unknown species (B52, B53, B55, B64).

Table 2 Production $\mathrm{H}_{2}$ and $\mathrm{CH}_{4}$ yields from Chlorella vulgaris and Dunaliella tertiolecta biomass after 49 days of incubation

\begin{tabular}{|c|c|c|c|c|}
\hline & $\mathrm{mmol} \mathrm{x}^{\mathrm{a}}$ per & $\begin{array}{l}\text { mmol } x^{a} \text { per } g \text { volatile } \\
\text { solids }\end{array}$ & $\begin{array}{l}\mathrm{mmol}^{\mathrm{a}} \text { per } \mathrm{g} \text { added } \\
\mathrm{COD}_{\text {tot }}\end{array}$ & $\begin{array}{l}\text { mmol xa per g removed } \\
\text { COD }_{\text {tot }}\end{array}$ \\
\hline \multicolumn{5}{|l|}{$\overline{\mathrm{CH}_{4}}$} \\
\hline C. vulgaris and $\mathrm{U}-\mathrm{C}$ & 59.6 & 11.9 & 5.8 & 11.3 \\
\hline C. vulgaris and $B-C$ & 0 & 0 & 0 & 0 \\
\hline D. tertiolecta and U-D & 5.1 & 1.0 & 2.1 & 3.6 \\
\hline D. tertiolecta and B-D & 0 & 0 & 0 & 0 \\
\hline \multicolumn{5}{|l|}{$\mathrm{H}_{2}$} \\
\hline C. vulgaris and no inoculum & 2.3 & 0.45 & 0.23 & 1.7 \\
\hline C. vulgaris, BESA and no inoculum & 1.6 & 0.33 & 0.15 & $-b^{b}$ \\
\hline D. tertiolecta and no inoculum & 2.6 & 0.52 & 1.6 & 21.1 \\
\hline $\begin{array}{l}\text { D. tertiolecta, BESA and no } \\
\text { inoculum }\end{array}$ & 1.7 & 0.35 & 0.42 & 4.0 \\
\hline
\end{tabular}

Where $\mathrm{x}$ is $\mathrm{CH}_{4}$ or $\mathrm{H}_{2}$, as indicated. ${ }^{\mathrm{b}} \mathrm{H}_{2}$ yield per $\mathrm{g}$ removed $\mathrm{COD}_{\text {tot }}$ could not be calculated as no $\mathrm{COD}_{\text {tot }}$ reduction was detected. $\mathrm{BESA}=2$-bromoethanesulfonic acid; $\mathrm{COD}_{\text {tot }}=$ total chemical oxygen demand. 


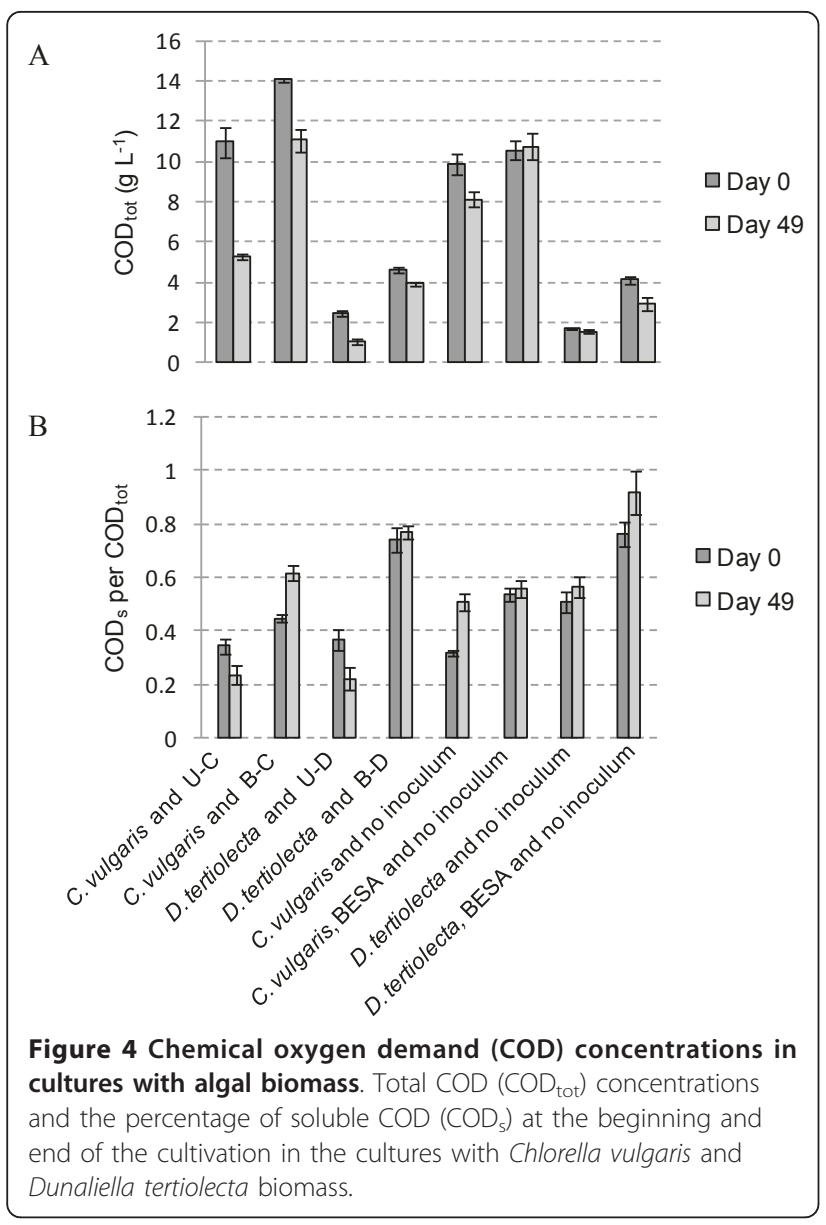

The DGGE profiles of bacteria associated with $C$. vulgaris and $D$. tertiolecta biomass were different. For example, $H$. alvei was seen only with $C$. vulgaris, whereas C. sulfidigenes and B. thermoamylovorans only with D. tertiolecta. In cultures with C. vulgaris, addition of BESA resulted in negligible changes in the bacterial DGGE profile. The only detectable difference was B54 that was identified from the cultures with BESA, but not in the cultures without BESA. In cultures with D. tertiolecta, bands B57, B58 and B67 were only visible in cultures without BESA and B63 was significantly brighter with BESA in the medium. Analysis of archaeal 16S rRNA gene sequences was not undertaken in this study.

\section{Discussion}

This work has demonstrated $\mathrm{CH}_{4}$ production from $C$. vulgaris and $D$. tertiolecta biomass when inoculated with municipal anaerobic digester sludge enrichments. Biogenic $\mathrm{H}_{2}$ was also produced, but it was subsequently consumed without $\mathrm{CH}_{4}$ production. $\mathrm{H}_{2}$ was produced also in the cultures with algal biomass but no anaerobic inoculum.
$\mathrm{H}_{2}$ was produced from both C. vulgaris and D. tertiolecta biomass by the $\mathrm{H}_{2}$ enrichment cultures (containing BESA), but it was subsequently consumed by nonmethanogenic microorganisms. The $\mathrm{pH}$ was relatively high in these assays. In the cultures with added anaerobic inoculum, $\mathrm{H}_{2}$ production was most sustained in the positive controls with glucose, where the $\mathrm{pH}$ was also the lowest. Karadag and Puhakka [18] showed with an anaerobic, moderately thermophilic $\left(45^{\circ} \mathrm{C}\right)$ enrichment culture that the $\mathrm{pH}$ significantly affected $\mathrm{H}_{2}$ production from glucose due to $\mathrm{pH}$ mediated shifts in fermentation pathways and the bacterial community composition. They reported $\mathrm{pH} 5.0$ was optimal for $\mathrm{H}_{2}$ production.

In the present work, several bacteria were identified from the anaerobic inoculum and algal biomass. These included Petrimonas spp. that have been previously shown to produce $\mathrm{H}_{2}$ [19]. Syntrophobacter spp. have been shown to convert propionate to acetate, $\mathrm{H}_{2}$ and $\mathrm{CO}_{2}$, but only when cocultivated with $\mathrm{H}_{2}$-consuming organisms $[20,21]$. B. wadsworthia and $W$. succinogenes utilize $\mathrm{H}_{2}$ as their electron donor [22,23]. According to Chassard et al. [24]Bacteroidetes spp. can suppress $\mathrm{H}_{2}$ production from cellulosic material in a mixed culture because they are non- $\mathrm{H}_{2}$-producing bacteria with a relatively high cellulolytic activity. O. indolifex is an obligately aerobic marine bacterium [25] with no activity under anaerobic conditions and thus it originated from the algal biomass slurry.

$\mathrm{H}_{2}$ accumulated in the cultures supplemented only with algal biomass. These cultures formed $\mathrm{CO}_{2}$ and accumulated organic acids and alcohols. Gfeller and Gibbs [15], Miura et al. [16] and Ueno et al. [17] reported hydrogen fermentation by microalgal cells under dark and anaerobic conditions, with $\mathrm{H}_{2}$ yields up to $2 \mathrm{mmol} \mathrm{H}_{2} / \mathrm{g}$ dry weight [16]. In this study, $\mathrm{H}_{2}$ yields were approximately $25 \%$ of that in the cultures with no added anaerobic inoculum (Table 2). However, the DGGE profiles had matches with several $\mathrm{H}_{2}$-producing bacteria such as Clostridium spp. [26,27] and Hafnia alvei [28], which are known $\mathrm{H}_{2}$ producers. Some Bacillus spp., such as B. cereus, B. thuringiensis [29] and B. megaterium [30] also produce $\mathrm{H}_{2}$. According to Combet-Blanc et al. [31], B. thermoamylovorans does not produce $\mathrm{H}_{2}$. O. proteus is typical in breweries and is known to cause beer spoilage [32]. Some Exiguobacterium spp. such as E. profundum are facultatively anaerobic and produce lactate as the main fermentation product [33].

Carver et al. [5] used the same algal biomass stocks but a different source inoculum to monitor metabolite production under thermophilic $\left(60^{\circ} \mathrm{C}\right)$ conditions. They reported $\mathrm{H}_{2}$ production without anaerobic inoculum by heterotrophs associated with C. vulgaris biomass, but low $\mathrm{H}_{2}$ production with heterotrophs associated with $D$. 


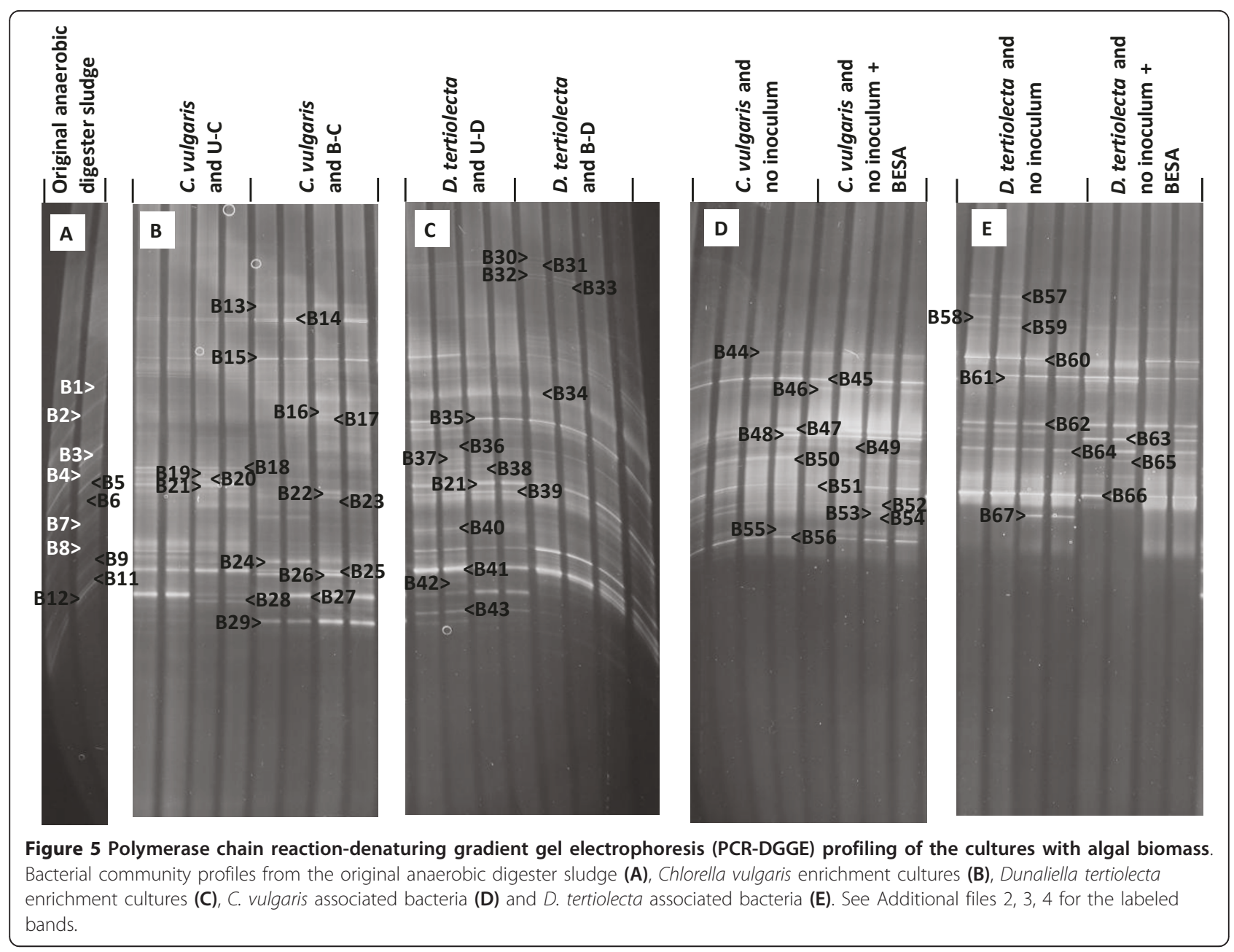

tertiolecta. In the present study, the D. tertiolecta-associated bacteria produced somewhat more $\mathrm{H}_{2}$, but approximately 4.5 times less VFA and alcohols and approximately 3 times less $\mathrm{CO}_{2}$ than the C. vulgarisassociated bacteria. The higher $\mathrm{H}_{2}$ production from $D$. tertiolecta was likely due to the lack of proper cell wall in $D$. tertiolecta and differences in bacterial composition of the algal biomass slurry. However, the $\mathrm{H}_{2}$ yields reported in this study were low. For comparison, Park et al. [34] reported the production of $28 \mathrm{ml} \mathrm{H}_{2}$ per g dry weight of the macroalga Laminaria japonica pretreated by ball milling and heat treatment at $120^{\circ} \mathrm{C}$ for $30 \mathrm{~min}$ using anaerobic sewage sludge as an inoculum. Carver et al. [5] reported production of 82 and $114 \mathrm{ml} \mathrm{H}_{2} / \mathrm{g} \mathrm{VS}$ from C. vulgaris and 39 and $58 \mathrm{ml} \mathrm{H}_{2} / g$ VS from D. tertiolecta by only microalgal associated bacteria and by a thermophilic consortium at $60^{\circ} \mathrm{C}$, respectively.

In the cultures with no added anaerobic inoculum, $\mathrm{H}_{2}$ production was somewhat lower with BESA in the medium. This indicates that BESA was inhibitory to some bacteria involved in fermentation. Bacteria present in cultures with no added anaerobic inoculum were associated with the algal culture or were introduced during handling of the biomass.

$\mathrm{CH}_{4}$ was produced from both $C$. vulgaris and D. tertiolecta biomass, but the yields were not comparable. $\mathrm{CH}_{4}$ production was approximately 12 times higher from $C$. vulgaris than from $D$. tertiolecta per added VS but only approximately 3 times higher per added or removed $\mathrm{COD}_{\text {tot }}$ (Table 2). Based on the chemical composition (protein, lipid and sugar content) of the two algal biomass feedstocks, theoretical $\mathrm{CH}_{4}$ yield according to Sialve et al. [35] would be 463 and $261 \mathrm{ml} \mathrm{CH}_{4} / \mathrm{g}$ VS from $C$. vulgaris and $D$. tertiolecta, respectively. The $\mathrm{CH}_{4}$ yields obtained (286 and $24 \mathrm{ml} \mathrm{CH}_{4} / \mathrm{g}$ VS) were $62 \%$ and $9 \%$ of the theoretical for C. vulgaris and D. tertiolecta, respectively. However, the cellular composition and major cellular fractions are greatly influenced by storage and culture conditions and cell age. Storage enhances cellular leakage, which was more pronounced with D. tertiolecta than with $C$. vulgaris. Based on Becker [36], C. vulgaris composition varies on average 
in the range of $51 \%$ to $58 \%$ protein, $14 \%$ to $22 \%$ lipids, and $2 \%$ to $17 \%$ carbohydrate on dry weight basis. Sydney et al. [37] reported $29 \%$ proteins, $11 \%$ lipids and $14 \%$ sugars for $D$. tertiolecta and the closely related $D$. salina contains $57 \%$ protein, $6 \%$ lipids, and $32 \%$ carbohydrate [36]. Additional file 1, Table S1 is a compilation of composition data pooled from specific studies; it is apparent that the bulk cellular composition is a variable parameter.

The large difference in $\mathrm{CH}_{4}$ production between the two algal biomasses was likely due to inhibition of digester sludge enrichment by the salinity in the marine $D$. tertiolecta slurry flocculated with $\mathrm{NaOH}[6,35,38]$. Salt toxicity towards methanogens is generally caused by the cation portion of the salt [38], which in this case is $\mathrm{Na}^{+}$. For example, McCarty [38] has reported 3.5 to $5.5 \mathrm{~g} / \mathrm{l}$ $\mathrm{Na}^{+}$to be moderately toxic and concentrations above 8 g/l highly toxic to methanogens. Similarly, high $\mathrm{Cl}^{-}$ levels can also cause inhibition of non-marine methanogens. The levels of dissolved $\mathrm{Na}^{+}$in cultures with $D$. tertiolecta in this study were $2.1 \mathrm{~g} / \mathrm{l}$ indicating non-toxic levels of $\mathrm{Na}^{+}$. However, $\mathrm{Cl}^{-}$concentration was significantly higher in cultures with $D$. tertiolecta than with $C$. vulgaris as the feedstock. It was also clearly seen from freeze-dried $D$. tertiolecta that salts were bound on the surface of the biomass. Similar salt precipitation was not seen in C. vulgaris biomass. Another reason for low $\mathrm{CH}_{4}$ production from $D$. tertiolecta biomass may be that $W$. succinogenes was identified from cultures with $D$. tertiolecta and U-D, but not from cultures with C. vulgaris and U-C. Coexistence of W. succinogenes has been reported to markedly reduce $\mathrm{CH}_{4}$ production [39].

Chen and Oswald [4] reported $320 \mathrm{ml} \mathrm{CH}_{4} / \mathrm{g}$ VS from biomass of a mixed microalgal culture from high-rate sewage stabilization ponds heat treated at $100^{\circ} \mathrm{C}$ for $8 \mathrm{~h}$. Yen and Brune [8] reported $143 \mathrm{ml} \mathrm{CH}_{4} / \mathrm{g}$ VS from an algal mixture including Scenedesmus spp. and Chlorella spp. without pretreatment. Thus, the $\mathrm{CH}_{4}$ yield achieved from C. vulgaris was comparable with previous results, but the yield from $D$. tertiolecta was very low. C. vulgaris biomass also contained some chitosan, used in flocculation of the biomass. Co-digestion of algal biomass (N-rich material) with $\mathrm{C}$-rich material such as cellulose or chitosan may enhance digestibility [8]. However, the anaerobic enrichments used in this study were not able to utilize chitosan very efficiently. Thus the co-digestion effect was negligible and $\mathrm{CH}_{4}$ was mainly produced from the algal biomass.

The calorific yields calculated for the maximum $\mathrm{H}_{2}$ and $\mathrm{CH}_{4}$ yields were $0.14 \mathrm{~kJ} / \mathrm{g}$ VS (for $\mathrm{H}_{2}$ production from $D$. tertiolecta without added anaerobic inoculum) and $10 \mathrm{~kJ} / \mathrm{g}$ VS (for $\mathrm{CH}_{4}$ production from C. vulgaris with enriched digester sludge without BESA). Hydrolytic pretreatment of algal slurries could substantially improve $\mathrm{H}_{2}$ production from complex biomass substrate. C. vulgaris biomass was shown to be amenable to methanogenic digestion without pretreatment, whilst the high salt content of $D$. tertiolecta biomass likely lowered the $\mathrm{CH}_{4}$ yields. However, based on $\mathrm{COD}_{\text {tot }}$, approximately $50 \%$ of $C$. vulgaris biomass was degraded during methanogenic fermentation. Therefore, pretreatment could also enhance $\mathrm{CH}_{4}$ production from the biomass of thick cell walled algae, such as $C$. vulgaris, but the energy cost of the pretreatment need to be considered.

\section{Conclusions}

$\mathrm{CH}_{4}$ was produced from C. vulgaris and D. tertiolecta biomass by mesophilic municipal anaerobic digester sludge enrichments. $\mathrm{H}_{2}$ was also produced with the anaerobic enrichments but was concurrently consumed by non-methanogenic microorganisms. $\mathrm{H}_{2}$ was produced by satellite bacteria associated with algal biomass. PCRDGGE profiling demonstrated the presence of $\mathrm{H}_{2}$ producing (for example, Petrimonas spp., Syntrophobacter spp.) and $\mathrm{H}_{2}$ consuming bacteria (for example, Bilophila wadsworthia, Wolinella succinogenes) in the anaerobic enrichments and $\mathrm{H}_{2}$ producing bacteria (for example, Clostridium spp., Hafnia alvei) among the satellite bacteria of both microalgal biomasses. $\mathrm{H}_{2}$ production by the satellite bacteria was comparable from $D$. tertiolecta and from $C$. vulgaris, but $\mathrm{CH}_{4}$ production by the anaerobic enrichments was substantially higher from C. vulgaris than from $D$. tertiolecta. The $\mathrm{CH}_{4}$ yield obtained from $D$. tertiolecta biomass with the inoculum originating from anaerobic digester was likely limited by the high salinity of the biomass, while the low protein, lipid and carbohydrate content of the D. tertiolecta further lowered the $\mathrm{CH}_{4}$ yield.

\section{Methods}

\section{Microalgal biomass production and harvest}

Chlorella vulgaris (Culture Collection of Algae and Protozoa, UK strain 211/11B) and Dunaliella tertiolecta (Sammlung von Algenkulturen Göttingen, Germany, strain SAG 13.86) were grown photoautotrophically in 201 column (diameter $0.16 \mathrm{~m}$ ) photobioreactors with $0.5 \mathrm{vvm}$ air sparging and photosynthetically active radiation at photon flux density averaging $225 \mu \mathrm{mol} / \mathrm{m}^{2} / \mathrm{s}$. C. vulgaris was grown in milliQ-water-based Jaworski's medium (http://www.ccap.ac.uk/media/recipes/JM.htm) and $D$. tertiolecta in natural seawater from the Menai Strait, UK, treated by filtration $(0.2 \mu \mathrm{m})$ and UV irradiation, with nutrients supplied according to Walne's medium (http://www.ccap.ac.uk/media/documents/Walnes. pdf).

Algal biomass was harvested from 201 cultures by flocculation followed by centrifugation. C. vulgaris was harvested by adding a chitosan stock solution (4 g 
chitosan, $50 \mathrm{ml}$ acetic acid, $950 \mathrm{ml}$ water) to the culture at approximately $2 \%$ of the total volume and adjusting $\mathrm{pH}$ to 7 by adding $3 \mathrm{M} \mathrm{NaOH}$ to initiate the flocculation. D. tertiolecta was flocculated by adding $50-100 \mathrm{ml}$ of $3 \mathrm{M} \mathrm{NaOH}$ to raise the $\mathrm{pH}$ to approximately $\mathrm{pH} 9.5$ [40]. The biomass of both species was then collected and centrifuged at $1,000 \mathrm{~g}$ for $10 \mathrm{~min}$ to produce a thick paste. The $\mathrm{pH}$ of $C$. vulgaris and $D$. tertiolecta biomass was adjusted to $7.0 \pm 0.2$ with $\mathrm{HCl}$ and the biomass slurries were stored at $-20^{\circ} \mathrm{C}$ until used in the gas production experiments. The algal biomass stocks were normalized by measurements of VS.

\section{Experimental conditions}

Anaerobic inocula were enriched from an anaerobic digester treating municipal wastewater sludge (City of Tampere, Finland). Serum bottle enrichments were prepared as series of batch incubations at $37^{\circ} \mathrm{C}$ with $5 \mathrm{~g}$ $\mathrm{VS} / \mathrm{l}$ of substrate. In the first three phases the substrate consisted of $25 \%$ (VS/VS) algal biomass and 75\% (VS/ VS) of activated sludge, followed by $50 \%$ of algal biomass and $50 \%$ of activated sludge, and finally $75 \%$ of algal biomass and $25 \%$ of activated sludge. In the following enrichment phases, $100 \%$ of algal biomass was used. Four different cultures were enriched. Two $\mathrm{H}_{2}$-fermenting cultures, one with $C$. vulgaris biomass, designated as B-C, and one with D. tertiolecta biomass as the substrate, B-D, and two $\mathrm{CH}_{4}$-producing cultures, one utilizing C. vulgaris biomass, U-C, and one D. tertiolecta biomass, U-D. Methanogenesis was suppressed in the $\mathrm{H}_{2}$-fermenting cultures by addition of $20 \mathrm{mM}$ BESA. The medium was prepared according to Zehnder et al. [41] with modifications by Karlsson et al. [42] and Ejlertsson et al. [43].

Gas production potential from C. vulgaris and D. tertiolecta was studied after nine passages of the corresponding enrichment culture at $37^{\circ} \mathrm{C}$ in $120 \mathrm{ml}$ anaerobic serum bottles with $50 \mathrm{ml}$ of medium and $10 \%$ (v/v) inoculum. The incubations included two types of negative controls, with inoculum but no substrate and with $5 \mathrm{~g}$ VS/l algal biomass but without anaerobic enrichment inoculum. Three types of positive controls were prepared containing enriched anaerobic inoculum and either $5 \mathrm{~g} / \mathrm{l}$ glucose, $5 \mathrm{~g} / \mathrm{l}$ cellulose or $5 \mathrm{~g} / \mathrm{l}$ chitosan.

\section{Chemical analyses}

The VS concentrations of the biomass samples were measured according to the Finnish Standard SFS 3008 [44]. Carbon and nitrogen were measured with ThermoElectron Flash EA 1112 after drying the samples at $80^{\circ} \mathrm{C}$ for 72 hours. The elemental analyzer was calibrated using the standards sulfanilamide, 2,5-bis(5'-tert-butylbenzoxazolyl)thiophene and l-cystine. dl-methionine was used as a reference material. Mass composition of the two microalgal biomass feedstocks was determined with analytical methods generally used in microalgal studies and at least three replicate samples were included in all analyses. The total lipid content of biomass was measured by extracting the lipids from freeze-dried biomass with chloroform/methanol and determining the lipids gravimetrically [45]. The protein composition of the algal biomass was calculated by multiplying the total elemental nitrogen content by 4.44 [46]. Total carbohydrate concentration of the biomass feedstocks was determined by the phenol sulfuric acid method [47]. Prior to biomass analyzes $D$. tertiolecta biomass was washed with $0.5 \mathrm{M}$ ammonium formate.

Gas production was measured according to Owen et al. [48]. The headspace gas composition $\left(\mathrm{H}_{2}, \mathrm{CH}_{4}\right.$ and $\mathrm{CO}_{2}$ ) was measured using Shimadzu gas chromatograph GC-2014 equipped with Porapak N column (80/100 mesh) and a thermal conductivity detector. The temperatures of the oven, injector and detector were 80, 110 and $110^{\circ} \mathrm{C}$, respectively. $\mathrm{N}_{2}$ was used as carrier gas at a flow rate of $20 \mathrm{ml} / \mathrm{min}$. The formation of organic acids and alcohol (lactate, formate, acetate, propionate, butyrate and ethanol) was analyzed with a Shimadzu HPLC chromatograph with a Shodex Sugar SH1011 column (Showa Denko K.K., Tokyo, Japan) and a refractive index detector (Shimadzu, Kyoto, Japan). Mobile phase was $5 \mathrm{mM} \mathrm{H}_{2} \mathrm{SO}_{4}$ and flow rate $0.9 \mathrm{ml} / \mathrm{min}$. COD was analyzed before $\left(\mathrm{COD}_{\text {tot }}\right)$ and after filtration $\left(\mathrm{COD}_{\mathrm{s}}\right)$ through $0.45 \mu \mathrm{m}$ polyester syringe filter (MachereyNagel, Düren, Germany) with dichromate method according to standard SFS 5504 [49]. Concentration of dissolved chloride ions was analyzed with Dionex DX120 ion chromatograph equipped with AS40 auto sampler and IonPac AS23 $(4 \times 250 \mathrm{~mm})$ anion exchange column. The mobile phase was $\mathrm{Na}$-carbonate/Na-bicarbonate solution containing $4.5 \mathrm{mM} / 1 \mathrm{Na}_{2} \mathrm{CO}_{3}$ and 3 $\mathrm{mM} / \mathrm{l} \mathrm{NaHCO}$. Concentration of dissolved sodium ions was analyzed with inductively coupled plasma emissionmass spectrometry according to industry standard DIN EN ISO 17294.

\section{Microbial community analyses}

Duplicate samples of $1.5 \mathrm{ml}$ were taken from the original digester sludge and from batch bottles at the end of the 49-day incubation and stored at $-20^{\circ} \mathrm{C}$. Prior to DNA extraction samples were pelleted by centrifugation $(10,000 \mathrm{~g}, 5 \mathrm{~min})$ and the supernatant removed. DNA was extracted from the pellets with PowerSoil DNA isolation kit (MO BIO Laboratories, Inc., Carlsbad, CA, USA). The extracted DNA sample was used as a template for the PCR. Partial bacterial 16S rRNA genes of the community DNA were amplified by using primer pair GC-BacV3f [50] and 907r [51] as described by Koskinen et al. [26]. DGGE was performed with 
INGENYphorU2 $\times 2$-system (Ingeny International BV, GP Goes, The Netherlands) using $8 \%$ polyacrylamide gels with denaturing gradient from $30 \%$ to $70 \%$ (100\% denaturing solution contains $7 \mathrm{M}$ of urea and $40 \%$ formamide). Gels were run at $60^{\circ} \mathrm{C}$ in $1 \times$ TAE ( $40 \mathrm{mM}$ Tris, $20 \mathrm{mM}$ acetic acid, $1 \mathrm{mM}$ ethylenediaminetetra-acetic acid (EDTA), pH 8.3) with $100 \mathrm{~V}$ for $22 \mathrm{~h}$ and stained with SYBR Gold (Molecular Probes Invitrogen, Eugene, OR, USA). The dominant bands were excised from the gels, eluted in $20 \mu \mathrm{l}$ of sterile water at $4^{\circ} \mathrm{C}$ overnight, stored at $-20^{\circ} \mathrm{C}$ and reamplified for sequencing. Sequencing was conducted at Macrogen Inc. (Seoul, Korea). Sequence data were analyzed with BioEdit software and compared with sequences in GenBank.

\section{Calculations}

Cumulative $\mathrm{H}_{2}$ and $\mathrm{CH}_{4}$ production were calculated according to Logan et al. [52]. The data were fitted to a modified Gompertz equation [53] by minimizing the square of the measurements and the estimates subtraction to give lag times and $\mathrm{H}_{2} / \mathrm{CH}_{4}$ production rates. The calorific yields from maximum $\mathrm{H}_{2}$ and $\mathrm{CH}_{4}$ yields were calculated from the lower heating values, $120 \mathrm{MJ} / \mathrm{kg}$ for $\mathrm{H}_{2}$ and $50 \mathrm{MJ} / \mathrm{kg}$ for $\mathrm{CH}_{4}$.

\section{Additional material}

Additional file 1: Mass composition of various microalgae. Mass composition (dry weight basis) data of microalgae pooled from literature sources.

Additional file 2: Bacterial band identities from the initial sludge. Matches of selected band identities of PCR-denaturing gradient gel electrophoresis (PCR-DGGE) samples from the initial anaerobic digester sludge.

Additional file 3: Bacterial band identities from the cultures with algal biomass and anaerobic enrichment inocula. Matches of selected band identities of PCR-denaturing gradient gel electrophoresis (PCRDGGE) samples from cultures with algal biomass and enriched anaerobic inocula.

Additional file 4: Bacterial band identities from the cultures with algal biomass and no anaerobic enrichments. Matches of selected band identities of PCR-denaturing gradient gel electrophoresis (PCRDGGE) samples from the cultures with algal biomass and no anaerobic inoculum.

\section{Acknowledgements}

This research was funded by the Finnish Funding Agency for Technology and Innovation (Finland Distinguished Professor Program, 402/06), Academy of Finland (Finland Distinguished Professor Program) and EPSRC (UK) Industrial Case Award with RWE nPower. We would like to thank Dr Elena Efimova for assistance with the lipid analyses.

\section{Author details}

'Department of Chemistry and Bioengineering, Tampere University of Technology, PO Box 541, Fl-33101 Tampere, Finland. 'School of Ocean Sciences, College of Natural Sciences, Bangor University, Menai Bridge, Anglesey LL59 5AB, UK. ${ }^{3}$ Finnish Environment Institute, Marine Centre, PO Box 140, Fl-00251 Helsinki, Finland. ${ }^{4}$ Department of Microbiology, Ohio State University, Columbus, OH 43210, USA.

\section{Authors' contributions}

AML carried out the anaerobic cultivations and all related analyses, microbial community analyses, data interpretation, and drafting and completion of the manuscript. CJH carried out microalgal biomass production and harvesting, and the elemental analysis of the harvested biomass and participated in the drafting of the manuscript. DNT participated in the design of microalgal biomass production and reviewed the manuscript. OHT participated in the design of the study and data interpretation, and thoroughly reviewed the manuscript. JAP conceived the study, participated in data interpretation and thoroughly reviewed the manuscript. All authors read and approved the final manuscript.

\section{Competing interests}

The authors declare that they have no competing interests.

Received: 31 January 2011 Accepted: 26 September 2011

Published: 26 September 2011

\section{References}

1. Schenk PM, Thomas-Hall SR, Stephens E, Marx UC, Mussgnug JH, Posten C, Kruse O, Hankamer B: Second generation biofuels: high-efficiency microalgae for biodiesel production. Bioenergy Res 2008, 1:20-43.

2. Posten C, Schaub G: Microalgae and terrestrial biomass as source for fuels - a process view. J Biotechnol 2009, 142:64-69.

3. Fan LT, Gharpuray MM, Lee YH: Evaluation and pretreatments for enzymatic conversion of agricultural residues. Biotechnol Bioeng Symp 1981, 11:29-45.

4. Chen PH, Oswald WJ: Thermochemical treatment for algal fermentation. Environ Int 1998, 24:889-897.

5. Carver SM, Hulatt CJ, Thomas DN, Tuovinen $\mathrm{OH}$ : Thermophilic, anaerobic co-digestion of microalgal biomass and cellulose for $\mathrm{H}_{2}$ production. Biodegradation 2011, 22:805-814.

6. Samson R, LeDuy A: Influence of mechanical and thermochemical pretreatments on anaerobic digestion of Spirulina maxima algal biomass. Biotechnol Lett 1983, 5:671-676.

7. Samson R, LeDuy A: Improved performance of anaerobic digestion of Spirulina maxima algal biomass by addition of carbon-rich wastes. Biotechnol Lett 1983, 5:677-682.

8. Yen HW, Brune DE: Anaerobic co-digestion of algal sludge and waste paper to produce methane. Bioresour Technol 2007, 98:130-134.

9. De Schamphelaire L, Verstraete W: Revival of the biological sunlight-tobiogas energy conversion system. Biotechnol Bioeng 2009, 103:296-304.

10. Kosourov S, Tsygankov A, Seiber M, Ghirardi ML: Sustained hydrogen production by Chlamydomonas reinhardtii: effects of culture parameters. Biotechnol Bioeng 2002, 78:731-740.

11. Chader S, Hacene H, Agathos SN: Study of hydrogen production by three strains of Chlorella isolated from the soil in the Algerian Sahara. Int J Hydrogen Energy 2009, 34:4941-4946.

12. Melis A: Green alga hydrogen production: progress, challenges and prospects. Int J Hydrogen Energy 2002, 27:1217-1228.

13. Benemann JR: Hydrogen production by microalgae. J Appl Phycol 2000, 12:291-300.

14. Ike A, Toda N, Tsuji N, Hirata K, Miyamoto K: Hydrogen photoproduction from $\mathrm{CO}_{2}$-fixing microalgal biomass: application of halotolerant photosynthetic bacteria. J Ferment Bioeng 1997, 84:606-609.

15. Gfeller RP, Gibbs M: Fermentative metabolism of Chlamydomonas reinhardtii. Plant Physiol 1984, 75:212-218.

16. Miura Y, Ohta S, Mano M, Miyamoto K: Isolation and characterization of a unicellular marine green alga exhibiting high activity in dark hydrogen production. Agr Biol Chem 1986, 50:2837-2844.

17. Ueno $Y$, Kurano N, Miyachi S: Ethanol production by dark fermentation in the marine green alga, Chlorococcum littorale. J Ferment Bioeng 1998, 86:38-43.

18. Karadag D, Puhakka JA: Direction of glucose fermentation towards hydrogen or ethanol production through on-line $\mathrm{pH}$ control. Int J Hydrogen Energy 2010, 35:10245-10251.

19. Grabowski A, Tindall BJ, Bardin V, Blanchet D, Jeanthon C: Petrimonas sulfuriphila gen. nov., sp. nov., a mesophilic fermentative bacterium isolated from a biodegraded oil reservoir. Int I System Evolut Microbiol 2005, 55:1113-1121. 
20. Boone DR, Bryant MP: Propionate-degrading bacterium, Syntrophobacterwolinii sp. nov. gen. nov., from methanogenic ecosystems. Appl Environ Microbiol 1980, 40:626-632.

21. de Bok FAM, Plugge CM, Stams AJM: Interspecies electron transfer in methanogenic propionate degrading consortia. Water Res 2004, 38:1368-1375.

22. da Silva SM, Venceslau SS, Fernandes CLV, Valente FMA, Pereira IAC: Hydrogen as an energy source for the human pathogen Bilophila wadsworthia. Antonie Van Leeuwenhoek 2008, 93:381-390.

23. Gross R, Simon J, Theis F, Kröger A: Two membrane anchors of Wolinella succinogenes hydrogenase and their function in fumarate and polysulfide respiration. Arch Microbiol 1998, 170:50-58.

24. Chassard C, Gaillard-Martinie B, Bernalier-Donadille A: Interaction between $\mathrm{H}_{2}$-producing and non- $\mathrm{H}_{2}$-producing cellulolytic bacteria from the human colon. FEMS Microbiol Lett 2005, 242:339-344.

25. Wagner-Döbler I, Rheims H, Felske A, El-Ghezal A, Flade-Schröder D, Laatsch H, Lang S, Pukall R, Tindall BJ: Oceanibulbus indolifex gen. nov., sp. nov., a North Sea alphaproteobacterium that produces bioactive metabolites. Int I System Evolut Microbiol 2004, 54:1177-1184.

26. Koskinen PEP, Kaksonen AH, Puhakka JA: The relationship between instability of $\mathrm{H}_{2}$ production and compositions of bacterial communities within a dark fermentation fluidized-bed bioreactor. Biotechnol Bioeng 2007, 97:742-758.

27. Hung C-H, Cheng C-H, Guan D-W, Wang S-T, Hsu S-C, Liang C-M, Lin C-Y: Interactions between Clostridium sp. and other facultative anaerobes in a self-formed granular sludge hydrogen-producing bioreactor. Int J Hydrogen Energy 2011, 36:8704-8711.

28. Podesta JJ, Gutiérrez-Navarro AM, Estrella CN, Esteso MA: Electrochemical measurement of trace concentrations of biological hydrogen produced by Enterobacteriaceae. Res Microbiol 1997, 148:87-93.

29. Porwal S, Kumar T, Lal S, Rani A, Kumar S, Cheema S, Purohit HJ, Sharma R, Patel SKS, Kalia VC: Hydrogen and polyhydroxybutyrate producing abilities of microbes from diverse habitats by dark fermentative process. Bioresour Technol 2008, 99:5444-5451.

30. Jeong T-Y, Cha G-C, Yeom SH, Choi SS: Comparison of hydrogen production by four representative hydrogen-producing bacteria. I Ind Eng Chem 2008, 14:333-337.

31. Combet-Blanc Y, Ollivier B, Streicher C, Patel BKC, Dwivedi PP, Pot B, Prensier G, Garcia JL: Bacillus thermoamylovorans sp. nov., a moderately thermophilic and amylolytic bacterium. Int J System Bacteriol 1995, 45:9-16.

32. Prest AG, Hammond JRM, Stewart GSAB: Biochemical and molecular characterization of Obesumbacterium proteus, a common contaminant of brewing yeasts. Appl Environ Microbiol 1994, 60:1635-1640.

33. Crapart S, Fardeau ML, Cayol JL, Thomas P, Sery C, Ollivier B, CombetBlanc Y: Exiguobacterium profundum sp. nov., a moderately thermophilic, lactic acid-producing bacterium isolated from a deep-sea hydrothermal vent. Int J System Evolut Microbiol 2007, 57:287-292.

34. Park J-I, Lee J, Sim SJ, Lee J-H: Production of hydrogen from marine macro-algae biomass using anaerobic sewage sludge microflora. Biotechnol Bioprocess Eng 2009, 14:307-315.

35. Sialve B, Bernet N, Bernard O: Anaerobic digestion of microalgae as a necessary step to make microalgal biodiesel sustainable. Biotechnol Adv 2009, 27:409-416.

36. Becker EW: Micro-algae as a source of protein. Biotechnol Adv 2007, 25:207-210.

37. Sydney EB, Sturm W, de Carvalho JC, Thomaz-Soccol V, Larroche C, Pandey A, Soccol CR: Potential carbon dioxide fixation by industrially important microalgae. Bioresour Technol 2010, 101:5892-5896.

38. McCarty PL: Anaerobic waste treatment fundamentals. Part three. Public Works 1964, 95:91-94.

39. Iwamoto M, Asanuma N, Hino T: Ability of Selenomonas ruminantium, Veillonella parvula and Wolinella succinogenes to reduce nitrate and nitrite with special reference to the suppression of ruminal methanogenesis. Anaerobe 2002, 8:209-215.

40. Horiuchi J, Ohba I, Tada K, Kobayashi M, Kanno T, Kishimoto M: Effective cell harvesting of the halotolerant microalga Dunaliella tertiolecta with pH control. J Biosci Bioeng 2003, 95:412-415.

41. Zehnder AJB, Huser BA, Brock TD, Wuhrmann K: Characterization of an acetate-decarboxylating, non-hydrogen-oxidizing methane bacterium. Arch Microbiol 1980, 124:1-11.
42. Karlsson A, Ejlertsson J, Nezirevic D, Svensson BH: Degradation of phenol under meso- and thermophilic, anaerobic conditions. Anaerobe 1999, 5:25-35.

43. Ejlertsson J, Johansson E, Karlsson A, Meyerson U, Svensson BH: Anaerobic degradation of xenobiotics by organisms from municipal solid waste under landfilling conditions. Antonie van Leeuwenhoek 1996, 69:67-74.

44. Finnish Standards Association: Determination of total residue and total fixed residue in water, sludge and sediment.[http://sales.sfs.fi/index.jsp? setLang $=1]$.

45. Bligh EG, Dyer WJ: A rapid method of total lipid extraction and purification. Can J Biochem Physiol 1959, 37:911-917.

46. López CVG, García MCC, Fernández FGA, Bustos CS, Chisti Y, Sevilla JMF: Protein measurements of microalgal and cyanobacterial biomass. Bioresour Technol 2010, 101:7587-7591.

47. Dubois M, Gilles KA, Hamilton JK, Rebers PA, Smith F: Colorimetric method for determination of sugars and related substances. Anal Chem 1956, 28:350-356.

48. Owen WF, Stuckey DC, Healy JB Jr, Young LY, McCarty PL: Bioassay for monitoring methane potential and anaerobic toxicity. Water Res 1979, 13:485-492.

49. Finnish Standards Association: Determination of chemical oxygen demand $(C O D \mathrm{Cr})$ in water with the closed tube method. Oxidation with dichromate.[http://sales.sfs.fi/index.jsp?setLang=1].

50. Muyzer G, De Waal EC, Uitterlinden AG: Profiling complex microbial populations by denaturing gradient gel electrophoresis analysis of polymerase chain reaction-amplified genes coding for $16 \mathrm{~S}$ rRNA. Appl Environ Microbiol 1993, 59:695-700.

51. Muyzer G, Hottenträger S, Teske A, Waver C: Denaturing gradient gel electrophoresis of PCR-amplified 16S rDNA - A new molecular approach to analyse the genetic diversity of mixed microbial communities. In Molecular Microbial Ecology Manual. Edited by: Akkermans ADL, van Elsas JD, De Bruijn F. Dordrecht, The Netherlands: Kluwer Academic Publishers; 1996:, 3.4.4/1-23..

52. Logan $\mathrm{BE}$, Oh SE, Kim IS, van Ginkel S: Biological hydrogen production measured in batch anaerobic respirometers. Environ Sci Technol 2002, 36:2530-2535

53. Chen C-C, Li C-Y, Lin M-C: Acid-base enrichment enhances anaerobic hydrogen production process. Appl Microbiol Biotechnol 2002, 58:224-228.

doi:10.1186/1754-6834-4-34

Cite this article as: Lakaniemi et al: Biogenic hydrogen and methane production from Chlorella vulgaris and Dunaliella tertiolecta biomass. Biotechnology for Biofuels 2011 4:34.

\section{Submit your next manuscript to BioMed Central and take full advantage of:}

- Convenient online submission

- Thorough peer review

- No space constraints or color figure charges

- Immediate publication on acceptance

- Inclusion in PubMed, CAS, Scopus and Google Scholar

- Research which is freely available for redistribution

Submit your manuscript at www.biomedcentral.com/submit
C Biomed Central 